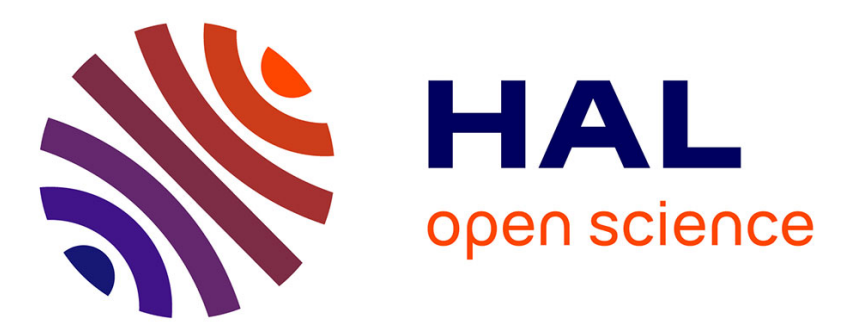

\title{
Les périodiques francophones dans le domaine information, communication et documentation : une étude empirique
}

\author{
Hélène Prost, Joachim Schöpfel
}

\section{- To cite this version:}

Hélène Prost, Joachim Schöpfel. Les périodiques francophones dans le domaine information, communication et documentation: une étude empirique. Documentaliste - Sciences de l'Information, 2012, 49 (3), pp.62-70. 10.3917/docsi.493.0062 . hal-00908592

\section{HAL Id: hal-00908592 \\ https://hal.science/hal-00908592}

Submitted on 25 Nov 2013

HAL is a multi-disciplinary open access archive for the deposit and dissemination of scientific research documents, whether they are published or not. The documents may come from teaching and research institutions in France or abroad, or from public or private research centers.
L'archive ouverte pluridisciplinaire HAL, est destinée au dépôt et à la diffusion de documents scientifiques de niveau recherche, publiés ou non, émanant des établissements d'enseignement et de recherche français ou étrangers, des laboratoires publics ou privés.

\section{(c)(1)}

Distributed under a Creative Commons Attribution| 4.0 International License 


\title{
Les périodiques francophones dans le domaine information, communication et documentation
}

\section{Une étude empirique}

\author{
Hélène Prost, Nancy \\ Joachim Schöpfel, Lille
}

\section{Résumé}

L'article dresse un panorama de l'offre des périodiques francophones dans le domaine information, communication et documentation (ICD) de l'année 2010. La constitution du corpus des 106 titres repose sur la compilation de onze sources différentes, associant bases de données, listes de référence et avis d'experts. L'image qui se dégage de notre analyse est contrastée. Le référencement des titres francophones renvoie l'image d'un domaine assez disparate, interdisciplinaire, transversal, dont les contours ne sont pas toujours clairs. Une autre caractéristique se définit par une forte contribution professionnelle mais sans réelle visibilité ni impact significatif sur la scène internationale, en dehors de la francophonie. La richesse des informations contenues dans le corpus permet cependant d'obtenir le reflet réaliste de l'état du domaine ICD. Sous l'angle de leurs périodiques, le domaine ICD se confirme comme une discipline composite, au carrefour entre recherche et métier, et fruit d'échanges constants avec d'autres disciplines.

\section{Introduction}

La revue scientifique joue un rôle central dans la communication scientifique, aussi bien pour la diffusion des résultats de recherche (production) que pour l'accès aux connaissances (consommation) [2].

Depuis plus de 60 ans, l'anglais est devenu la lingua franca des revues scientifiques [8]. Neuf revues sur dix sont publiées en anglais ${ }^{1}$. Au fil des années, l'anglais a relégué la plupart des autres langues à un statut régional ou marginal. Publier en français, allemand, espagnol réduit la visibilité et l'impact des résultats de recherche sur la scène internationale et nuit au classement des universités [25]. ${ }^{2}$ La surreprésentation des titres anglophones dans les outils scientométriques Web of Science et Scopus induit un biais dans toute évaluation.

Déjà en 2002, Garfield [9] remarqua que “(...) there is a great need for national journals written in German and other European languages, but it is absurd to expect them to reach the same level of impact as international journals, unless they adhere to the same standards as leading journals and attract significant original research.",

Cependant, aujourd'hui, la plupart des chercheurs français en sciences humaines et sociales publient leurs résultats de préférence en français et dans des revues francophones.

\footnotetext{
${ }^{1}$ Source : CSA Proquest Ulrichsweb.

${ }^{2}$ Cf le Leiden ranking : http://socialsciences.leiden.edu/cwts/products-services/leiden-ranking-2010-cwts.html

${ }^{3}$ «On a vraiment besoin de revues nationales en allemand ou en d'autres langues européennes mais il est absurde d'attendre qu'elles aient le même niveau d'impact que les revues internationales à moins qu'elles adoptent les mêmes règles que les titres phares et attirent d'importants travaux de recherche originaux » (traduction auteurs).
} 
Une étude du GFII constata en 2009 que « la publication en français est encore la règle ce qui pose le problème de la visibilité de la recherche française en SHS à l'international » [10].

Par ailleurs, ces documents restent une source d'accès privilégiée à l'information scientifique. "Les revues SHS, avant d'être l'expression du travail collectif d'une communauté disciplinaire ou sous-disciplinaire sont l'expression, le vecteur de communication d'équipes de recherche. Il en résulte un extrême morcellement de l'offre de revues SHS en France. » (ibid.)

Il est donc légitime de s'interroger sur les caractéristiques de ces périodiques, aussi bien sous l'aspect de l'évaluation que de la discipline.

Concrètement, cela signifie: Comment ces périodiques sont-ils référencés par les outils scientométriques et bibliométriques, comment sont-ils positionnés sur le marché de l'édition scientifique, comment assurent-ils une qualité minimale de leur contenu ? Et deuxièmement, quels disciplines et domaines scientifiques couvrent-ils, même d'une manière marginale ?

\section{Etat de l'art}

Nous avons choisi le domaine information, communication et documentation (ICD) ${ }^{4}$ comme champ d'investigation pour son caractère interdisciplinaire, sa proximité avec le milieu professionnel [17] et sa courte histoire [3] qui incite les chercheurs, plus encore que dans d'autres domaines, à veiller sur l'appartenance, le «bornage » [7] et les caractéristiques de leurs revues. Depuis quelques années, les revues ICD ont fait l'objet de plusieurs études :

Schloegl et Stock [21] ont associé une analyse des citations et une enquête de satisfaction pour répondre à deux questions: Quel est l'impact de 50 revues ICD anglophones et allemandes, et quels sont les titres les plus importants et visibles ? Comment les lecteurs utilisent-ils ces revues, comment ces revues sont-elles appréciées ? La méthodologie mixte produit plusieurs «ranking lists » avec en tête des titres comme Journal of Documentation, JASIST ou Bibliotheksdienst.

Schloegl et Petschnig [22] ont mené une enquête auprès des rédactions de 50 revues ICD anglophones et allemandes, afin de mieux connaitre ce secteur du marché éditorial. Le résultat est contrasté :

- Le marché est relativement concentré : $60 \%$ des revues sont publiées par sept éditeurs dont Emerald (GB) et Elsevier (NL).

- Le lectorat est composé de professionnels (50\%), chercheurs (33\%), industriels et autres $(17 \%)$.

- Le peer review est pratiqué pour deux tiers des titres (dont la moitié en aveugle). Le taux de rejets moyen est de 15-20\% mais dépasse parfois 50\%. Le délai de publication varie d'un mois à un an ou plus, avec une moyenne de six mois.

- Ambition et audience sont souvent nationales. Peu de comités de lecture ont un caractère international.

\footnotetext{
${ }^{4}$ Nous avons préféré le terme « domaine ICD » à celui des « sciences de l'information et de la communication » (SIC) car il correspond mieux au classement des bases de données (library and information sciences,

communication). Par la suite, « domaine ICD » ou « ICD » englobera SIC, bibliothéconomie et documentation. C'est un choix pragmatique et ICD est utilisé comme terme générique. L'article ne porte pas sur une définition disciplinaire mais sur les revues qui couvrent les thématiques ICD.
} 
Zhao [26] a analysé sept revues ICD sous l'aspect économique, mettant en rapport l'analyse des citations (impact) et le financement des projets. L'étude distingue entre information-oriented journals (recherche) et library-focused journals (métier).

$\mathrm{Hu}$ et al. [12] publient une analyse des co-citations de 24 revues ICD en Chine. L'analyse statistique permet d'identifier quatre groupes (clusters) qui se distinguent selon le domaine (science de l'information vs. bibliothéconomie) et l'audience (recherche vs. métier).

Bar-Ilan [1] propose un classement de 37 revues ICD à partir de leur h-index et du facteur d'impact du Web of Science. Arrivent en tête, MIS Quarterly et le Journal of the American Medical Informatics Association, suivis par des titres comme Information \& Management ou Scientometrics. Toutes sont publiées en langue anglaise.

En France, sous l'égide de l'ISCC (Institut des Sciences de la Communication du CNRS), le séminaire Valorisation des revues en sciences de l'information et de la communication, est créé en février 2008. Son objectif est d'initier une réflexion, parmi les acteurs opérationnels, sur le processus de publication, d'édition et de signalement de la production des chercheurs. Ses activités se concentrent sur la production d'enquêtes et l'organisation de deux journées d'étude annuelles, en 2008 puis 2009. ${ }^{5}$ [16]

Parmi ces enquêtes, celle de Jeannin [13] [14] applique une méthode dite " revuemétrie », définie comme «repérage scientifique (...) pour valoriser (les) publications (des chercheurs en sciences humaines et sociales) ». Il a ainsi identifié plusieurs centaines de revues dans huit disciplines SHS, dont 281 revues dans le domaine ICD publiées en 24 pays.

Rasse et al. [20] ont mené une enquête auprès d'enseignants-chercheurs et chercheurs afin d'identifier les revues qu'ils lisent, parmi un panel de 31 titres SIC francophones. En moyenne, les personnes interrogées utilisent régulièrement 3,3 revues auxquelles s'ajoutent quatre revues qu'elles consultent régulièrement. Il existe un lien fort entre les revues et leurs auteurs : $30 \%$ des répondants qui lisent régulièrement une revue publient aussi dans cette revue ; inversement $68 \%$ lisent les revues dans lesquelles ils publient. Face à l'internalisation de la discipline, Rasse insiste sur la «nécessité absolue de maintenir et de développer un « cœur » de revues francophones de la discipline.

De 2008 à 2010, Dassa et al. [6] ont recensé les périodiques en SHS dans les bases de données du Web of Science et Scopus ainsi que dans les listes d'autorité du European Reference Index for the Humanities (ERIH) et de l'AERES. La plateforme JournalBase ${ }^{6}$ détaille la représentativité des titres dans 27 domaines SHS et contient 286 revues en sciences de la communication.

Dans une étude récente sur les revues en SIC, Heinderyckx et al. [11] soulignent l'émergence de nouvelles formes de publication.

Quel est le point commun de ces études ? D'une manière générale, elles contribuent à une meilleure connaissance des revues ICD comme vecteur de communication scientifique et outil d'évaluation. Plus concrètement, elles aident à délimiter le champ disciplinaire et à consolider la cohérence de la communauté scientifique, par l'identification des établissements, auteurs, co-auteurs, références citées, réseaux etc. Par ailleurs, elles témoignent aussi de la réflexivité du domaine ICD et des regards croisés entre chercheur et professionnel de l'information.

Notre article poursuit le travail de ces études, notamment de Jeannin, Rasse et Dassa. Il dresse un panorama de l'offre de périodiques francophones ICD en 2010, dans une double perspective scientifique et professionnelle. Il s'intéresse à trois aspects :

\footnotetext{
${ }^{5}$ http://www.iscc.cnrs.fr/spip.php?article717\#outil_sommaire_0

${ }^{6} \underline{\text { http://journalbase.cnrs.fr/ }}$
} 
1. Leur classement thématique et/ou disciplinaire.

2. Leur visibilité internationale.

3. Leur facteur d'impact (Web of Science et Scopus).

La discussion portera sur la méthode d'analyse et sur d'éventuelles conséquences aussi bien pour l'édition que pour l'évaluation, la publication et la recherche.

\section{Méthodologie}

L'étude s'appuie sur une méthodologie développée par Dassa et al. [6] puis reprise par l'Institut de l'Information Scientifique et Technique du CNRS (INIST) dans le cadre d'un outil de visualisation ${ }^{7}$ de revues SHS à partir de plusieurs sources : les bases de données Arts \& Humanities Citation Index (AHCI) et Social Sciences Citation Index (SSCI) du Web of Science (WoS), Scopus, FRANCIS, les listes de l'AERES et de l'ERIH, les portails Revues.org et CAIRN. A ces sources, nous avons ajouté trois autres corpus : l'enquête de Jeannin [14], les Library and Information Science Abstracts (LISA) et le Ulrichsweb Global Serials Directory de ProQuest, la plus exhaustive base de périodiques contenant plus de 300000 titres dont 27000 revues scientifiques ${ }^{8}$. Voici les différentes sources caractérisées par leur critère d'extraction (indexation thématique dans le domaine ICD) et le nombre de revues ainsi obtenues :

\begin{tabular}{|l|c|l|c|}
\hline Source & Date d'extraction & Critère d'extraction & Nb titres \\
\hline AHCI $($ WoS $)$ & févr-10 & Information science \& library science & 8 \\
\hline SSCI (WoS) & févr-10 & Information science \& library science & 5 \\
\hline Scopus & déc-10 & $\begin{array}{l}\text { Library Information Science, Communication, Information System, } \\
\text { Information System Management, Media Technologies }\end{array}$ & 14 \\
\hline Ulrichsweb & déc-10 & $\begin{array}{l}\text { Library Information Science, Communication, Information Science } \\
\text { and Information Theory }\end{array}$ & 83 \\
\hline FRANCIS & févr-10 & Sciences de l'information & 41 \\
\hline ERIH & $2007-2008$ & Communication sciences & 10 \\
\hline Revues.org & mai-10 & Information & 12 \\
\hline CAIRN & mai-10 & Sc.info./communication & 15 \\
\hline AERES & oct-10 & Liste SIC rang A (section 71 CNU) & 37 \\
\hline Jeannin & $2000-2001$ & Revues en sciences de l'information et de la communication & 67 \\
\hline LISA & avr-12 & Serials source list (= library and information science) & 5 \\
\hline
\end{tabular}

Tableau 1 : Composition du corpus des revues ICD francophones (total 106 revues)

Pour notre étude, nous avons retenu toutes les revues indexées ICD par au moins une source et éditées en France et/ou de langue française. Cette démarche implique par exemple qu'une revue de médiation culturelle se trouve dans le corpus uniquement si elle a été indexée en SIC, LIS etc. - Si besoin, les informations bibliographiques ont été complétées avec le SUDOC, ISSN-Online, Google Scholar ou Scirus.

Pour finir, trois remarques : Nous n'avons pas analysé titre par titre. L'objet de notre étude est leur description et leur classement par des outils de sélection, d'évaluation et de recherche.

En particulier, nous n'avons pas évalué la scientificité des revues. Toutes les sources utilisées (WoS, Scopus etc.) ont fait une sélection à partir de leur caractère scientifique et/ou leur intérêt pour les chercheurs. L'objectif de notre étude n'est pas l'analyse de leurs critères de sélection. En conséquence, environ un tiers du corpus se rapproche de la presse spécialisée (magazines, bulletins, newsletters etc.) même si on y trouve aussi du contenu avec un intérêt scientifique.

\footnotetext{
${ }^{7}$ L'outil de visualisation est disponible sur le site de l'INIST http://www.inist.fr/spip.php?article85

${ }^{8}$ Scientifique veut dire ici, indexé comme scholarly ou academic.
} 
Nous n'avons pas non plus interrogé l'indexation thématique des différentes sources. Les SIC françaises ne correspondent pas tout à fait aux library and information sciences anglaises, américaines ou allemandes. Mais pour étudier les revues SIC francophones à partir de sources internationales, il faut accepter une certaine hétérogénéité. Pour éviter tout malentendu, nous avons donc systématiquement remplacé le terme SIC par « domaine ICD » (cf. aussi note 4).

\section{Résultats}

Les 106 revues de notre corpus sont publiées dans six pays différents, dont $77 \%$ en France et $12 \%$ au Canada (cf. tableau 2).

\begin{tabular}{|c|c|c|}
\hline Pays d'édition & Total & $\%$ \\
\hline France & 82 & $77 \%$ \\
\hline Canada & 13 & $12 \%$ \\
\hline Belgique & 7 & $6 \%$ \\
\hline Tunisie & 2 & $2 \%$ \\
\hline Algérie & 1 & $1 \%$ \\
\hline Suisse & 1 & $1 \%$ \\
\hline
\end{tabular}

Tableau 2 : Pays d'édition

Cent éditeurs différents se partagent le corpus francophone, parmi lesquels une grande majorité (94\%) publie une unique revue. Avec cinq titres, Lavoisier est l'éditeur le mieux représenté, suivi par le CNRS, A Jour, Armand Colin, l'ABF et L'Harmattan. 26 revues sont publiées par une université ou école, 17 autres par une association ou société savante.

Le rythme de parution de la moitié des revues (49) les rapproche des monographies en séries, car leur périodicité est soit annuelle, semestrielle ou irrégulière (tableau 3). Autant de revues (47) paraissent entre trois et six fois par an, tandis que dix périodiques sont publiés avec sept à douze numéros par an, se rapprochant de la presse spécialisée (magazines).

\begin{tabular}{|l|c|}
\hline Périodicité & Total \\
\hline Irrégulière, indéterminée & 7 \\
\hline $1-2$ fois/an & 42 \\
\hline $3-4$ fois/an & 40 \\
\hline $5-6$ fois/an & 7 \\
\hline $7-12$ fois/an & 10 \\
\hline
\end{tabular}

Tableau 3 : Périodicité

Comme le corpus a été élaboré à partir de sources extraites à des dates différentes, au moment de l'analyse (juillet 2011), 10 titres sont en cessation ou suspendus. Deux autres revues ont changé de titre.

\section{Le cœur et la périphérie}

Pour chaque revue du corpus nous avons comparé l'indexation thématique de trois sources françaises (FRANCIS, AERES, Jeannin) et quatre sources internationales (WoS, Scopus, LISA, Ulrichsweb).

Premier résultat : parmi les 106 titres du corpus, il n'y a pas un seul qui soit indexé ICD 9 par l'ensemble des sept sources. Une seule revue - Documentaliste-Sciences de l'information - fait consensus pour six sources, une autre - Canadian Journal of Information and Library Science - par cinq sources. Trois titres sont indexés ICD par quatre sources: Argus, Documentation et bibliothèques et Etudes de Communication, 14 autres par trois sources.

\footnotetext{
${ }^{9}$ Indexation ICD veut dire: sciences de l'information et de la communication, library and information sciences, communications, information science and information theory.
} 
Ensemble, ces titres correspondent à $18 \%$ du corpus (tableau 4).

\begin{tabular}{|l|l|l|}
\hline Archives (Québec, PQ) & Association des Archivistes du Québec & CA \\
\hline Argus & Corporation des Bibliothecaires Professionnels du Québec & CA \\
\hline $\begin{array}{l}\text { Canadian Journal of Information and Library } \\
\text { Science }\end{array}$ & University of Toronto Press & CA \\
\hline Communication (Québec) & Editions Nota Bene & CA \\
\hline Documentation et bibliothèques & $\begin{array}{l}\text { Association pour l'Avancement des Sciences et des } \\
\text { Techniques de la Documentation }\end{array}$ & CA \\
\hline Archimag & I D P s.a.r.l. & FR \\
\hline Bases (Paris) & Bases Publications & FR \\
\hline Bibliotheques de France. Bulletin & $\begin{array}{l}\text { Ecole nationale supérieure des sciences de l'information et des } \\
\text { bibliothèques ENSSIB }\end{array}$ & FR \\
\hline Communication et langages & Armand Colin & FR \\
\hline Communications \& stratégies & Institut de l'Audiovisuel et des Telecommunications en Europe & FR \\
\hline Document numérique & Lavoisier & FR \\
\hline Documentaliste & $\begin{array}{l}\text { Association française des documentalistes et des } \\
\text { bibliothécaires spécialisés }\end{array}$ & FR \\
\hline Etudes de Communication & Laboratoire GERIICO - Université de Lille 3 & FR \\
\hline La Revue des revues & Association Ent'revues & FR \\
\hline Le Temps Des Médias & Nouveau Monde Editions & FR \\
\hline MEI. Media et information & Université Paris VIII, département Infocom -- L'Harmattan & FR \\
\hline Mots (Paris. 1980) & E N S Editions & FR \\
\hline Réseaux: communication technologie societe & La Découverte & FR \\
\hline Revue tunisienne de communication & Institut de Presse et des Sciences de l'Information (IPSI) \\
\hline
\end{tabular}

Tableau 4 : Revues cœurs ICD (N=19)

Pour la description de ces titres, il existe apparemment un certain consensus, une convergence ou concordance d'indexation de plusieurs sources différentes. Peut-être pourraiton les considérer comme des revues cœurs dans le domaine en question, du moins d'une manière empirique.

Revenons au corpus des 106 titres. Du fait du caractère transdisciplinaire du domaine ICD, une partie significative est également indexée dans une autre discipline. Par exemple, Communication, Communication et langages et Etudes de Communication sont également indexées comme revues en sociologie ; La Revues des revues comme titre d'histoire; et Mots comme appartenant aussi à la sociologie et la linguistique.

L'analyse de l'indexation des disciplines limitrophes dégage trois catégories de disciplines, en fonction du nombre d'indexation :

- Trois disciplines limitrophes importantes : la sociologie avec l'anthropologie, la linguistique et l'histoire (avec les sciences politiques).

- Huit disciplines de moindre importance : l'informatique, les sciences de l'éducation, l'économie, les médias, la littérature, la philosophie et le droit, puis les SHS comme domaine général et multidisciplinaire.

- Cinq disciplines ou domaines en marge: l'art, la géographie, la psychologie, la théologie et la science comme domaine général et multidisciplinaire. 


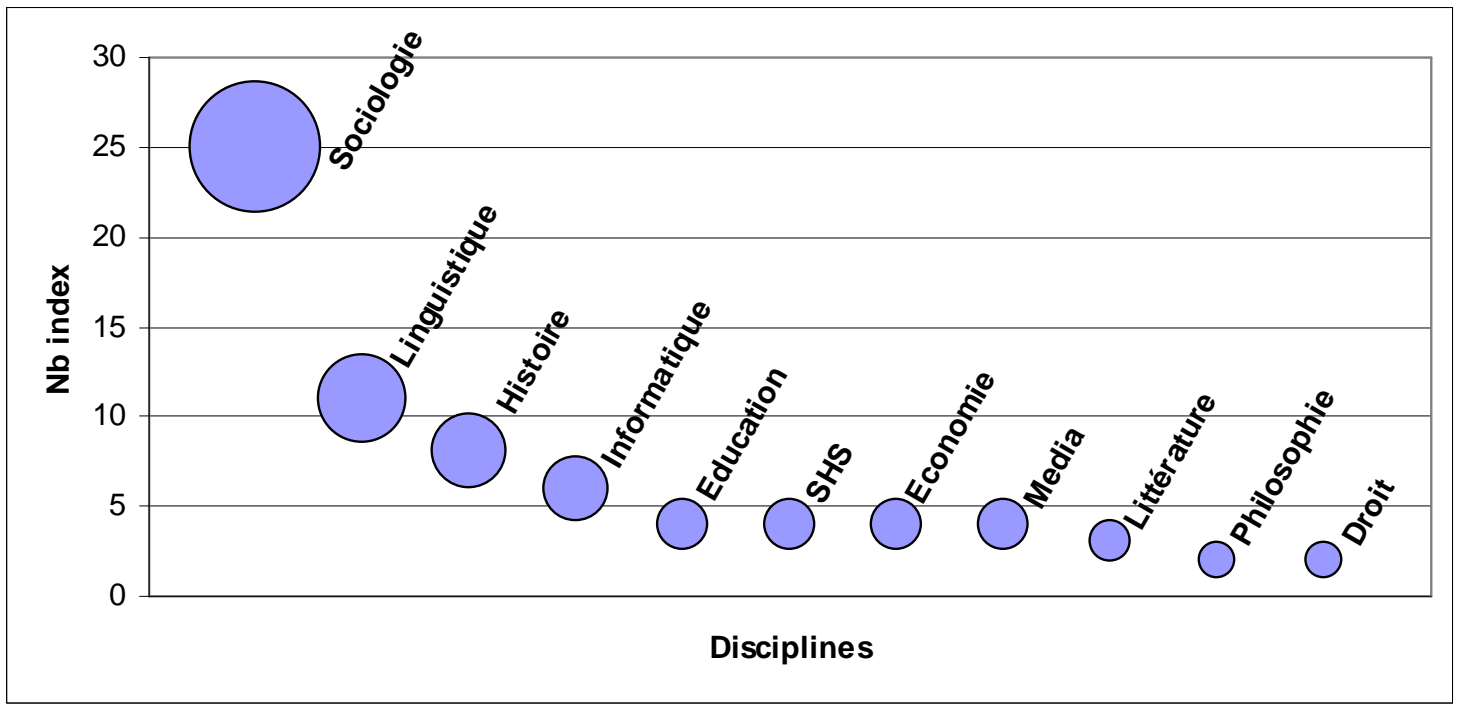

Figure 1 : Disciplines limitrophes

A partir des deux notions - revues cœurs et disciplines limitrophes - on pourrait établir une cartographie des revues ICD, sous forme de cercles concentriques et avec des facettes disciplinaires où l'appartenance à telle ou telle facette serait le reflet du caractère interdisciplinaire de la revue et/ou l'expression de l'échange entre les ICD et d'autres disciplines. Face à la dynamique des politiques scientifiques et au développement des revues en libre accès, on peut penser qu'il ne s'agit là que d'une photo instantanée, amenée à changer dans les années à venir.

\section{La visibilité internationale}

Comment les périodiques du corpus ICD sont-ils signalés sur le plan international ? Quelle est leur visibilité ? Pour avoir une idée, nous avons étudié leur indexation par Scopus, la plus importante base de données scientifique du moment, par LISA, une base thématique renommée, et par Ulrichsweb, la base de revues la plus exhaustive pour la gestion d'une collection.

LISA contient cinq titres francophones. Scopus indexe cinq titres de notre corpus dans les catégories library and information sciences (2), communication (2) ou les deux (1). De son côté, Ulrichsweb signale 18 titres en library and information sciences, 11 en communication et un en information science and information theory, en tout 30 titres. Sans les titres morts ou suspendus, il reste 27 revues (cf. tableau 5). 


\begin{tabular}{|c|c|c|c|c|c|}
\hline Titre & Editeur & Pays & Ulrichsweb & SCOPUS & LISA \\
\hline Alsic & Université de Strasbourg 2 & FR & Com & & \\
\hline Annales des Telecommunications & Springer France & FR & Com & Autre & \\
\hline Arbido & Staempfli Verlag AG & $\mathrm{CH}$ & Lis & & \\
\hline Archimag & I D P s.a.r.l. & FR & Lis & & \\
\hline Archivaria & $\begin{array}{l}\text { Association of Canadian } \\
\text { Archivists }\end{array}$ & CA & Lis & & Lis \\
\hline Archives (Québec, PQ) & $\begin{array}{l}\text { Association des Archivistes du } \\
\text { Québec }\end{array}$ & $\mathrm{CA}$ & Lis & & Lis \\
\hline Archives et Bibliotheques de Belgique & $\begin{array}{l}\text { Archives et Bibliothèques de } \\
\text { Belgique a.s.b.l. }\end{array}$ & BE & Lis & & \\
\hline Argus & $\begin{array}{l}\text { Corporation des Bibliothecaires } \\
\text { Professionnels du Québec }\end{array}$ & CA & Lis & & \\
\hline Bases (Paris) & Bases Publications & FR & Lis & & \\
\hline Bibliotheques de France. Bulletin & $\begin{array}{l}\text { Ecole nationale supérieure des } \\
\text { sciences de l'information et des } \\
\text { bibliothèques ENSSIB }\end{array}$ & FR & Lis & & Lis \\
\hline Cahiers de la Documentation & $\begin{array}{l}\text { Association Belge de } \\
\text { Documentation }\end{array}$ & BE & Lis & & \\
\hline Canadian Journal of Film Studies & $\begin{array}{l}\text { Film Studies Association of } \\
\text { Canada }\end{array}$ & CA & Com & & \\
\hline $\begin{array}{l}\text { Canadian Journal of Information and Library } \\
\text { Science }\end{array}$ & University of Toronto Press & CA & Lis & & Lis \\
\hline CinémAction & Editions Corlet S.A. & FR & Com & & \\
\hline Communications \& stratégies & $\begin{array}{l}\text { Institut de l'Audiovisuel et des } \\
\text { Telecommunications en Europe }\end{array}$ & FR & Com & & \\
\hline Document numérique & Lavoisier & FR & Autre & Lis & \\
\hline Documentaliste & $\begin{array}{l}\text { Association française des } \\
\text { documentalistes et des } \\
\text { bibliothécaires spécialisés }\end{array}$ & FR & Lis & Lis, Com & Lis \\
\hline Documentation et bibliothèques & $\begin{array}{l}\text { Association pour l'Avancement } \\
\text { des Sciences et des Techniques } \\
\text { de la Documentation }\end{array}$ & $\mathrm{CA}$ & Lis & & \\
\hline Etudes de Communication & $\begin{array}{l}\text { Laboratoire GERIICO - } \\
\text { Université de Lille } 3\end{array}$ & FR & Com & & \\
\hline Information interaction intelligence & Cepadues Editions & FR & Lis & & \\
\hline $\begin{array}{l}\text { International Journal of Information Sciences for } \\
\text { Decision Making }\end{array}$ & $\begin{array}{l}\text { Centre de Recherche } \\
\text { Retrospective de Marseille } \\
\end{array}$ & FR & Info & & \\
\hline Le Temps Des Médias & Nouveau Monde Editions & FR & Com & Com & \\
\hline $\begin{array}{l}\text { Partnership: the Canadian journal of library and } \\
\text { information practice and research }\end{array}$ & $\begin{array}{l}\text { The Partnership. Provincial and } \\
\text { Territorial Library Association of } \\
\text { Canada }\end{array}$ & $\mathrm{CA}$ & Lis & & \\
\hline Reseaux: communication technologie societe & La Découverte & FR & Autre & Com & \\
\hline Revue de la BNF & $\begin{array}{l}\text { Bibliothèque Nationale de } \\
\text { France }\end{array}$ & FR & Lis & & \\
\hline Revue tunisienne de communication & \begin{tabular}{|l|} 
Institut de Presse et des \\
Sciences de l'Information (IPSI)
\end{tabular} & TN & Com & & \\
\hline Scriptorium & Centre d'Etude des Manuscrits & BE & Autre & Lis & \\
\hline
\end{tabular}

Tableau 5 : Revues francophones indexés ICD dans Scopus, Ulrichsweb et LISA

Autrement dit, $25 \%$ des revues du corpus jouissent d'une certaine visibilité internationale. Ces titres sont publiés par 26 éditeurs de cinq pays différents. Le paysage est éclaté - neuf titres appartiennent à des éditeurs commerciaux (dont Lavoisier, Springer et Serda), neuf sont publiés par des sociétés savantes ou associations professionnelles, et neuf sont édités par des établissements de l'enseignement supérieur ou de la recherche (universités, écoles, laboratoires, instituts). Aucun acteur ne domine l'offre éditoriale. Plus de $3 / 4$ de ces titres existent en version électronique. ALSIC, BBF, ISDM et la revue Information Interaction Intelligence sont des titres en «open access » et sont répertoriés dans l'annuaire international 
DOAJ. Documentaliste et Etudes de Communication sont partiellement en libre accès mais pratiquent un embargo sur les articles récents.

Une dernière remarque concerne la visibilité internationale des revues considérées par l'AERES, FRANCIS ou Jeannin comme «essentielles » ou « cœur du domaine » des SIC. Un tiers seulement de ces titres sont indexés par Scopus, Ulrichsweb ou LISA (cf. tableau 6).

\begin{tabular}{|r|c|c|c|}
\hline Corpus initial & Nb titres vivants & $\begin{array}{c}\text { indexés dans } \\
\text { SCOPUS ou }\end{array}$ & En \% \\
\hline Jeannin & 52 & 16 & $31 \%$ \\
\hline FRANCIS & 41 & 13 & $32 \%$ \\
\hline AERES & 37 & 10 & $27 \%$ \\
\hline
\end{tabular}

Tableau 6 : Visibilité internationale des revues francophones SIC

Deux tiers des titres jugés par la communauté scientifique comme de bonne ou très bonne qualité manquent donc de visibilité internationale.

\section{Qualité et impact}

Malgré une critique constante sur la fiabilité, l'objectivité et l'application, le nombre des citations est toujours interprété comme indice de la qualité d'une revue. Aujourd'hui, il existe surtout deux indicateurs calculés à partir des citations, le facteur d'impact de l'Institut of Scientific Information ISI (Journal Citation Reports JCR du WoS) et celui de l'équipe espagnole SCImago (SCImago Journal Rank Indicator SJR de Scopus). ${ }^{10}$ Comment se situent les revues francophones ICD par rapport à ces deux indicateurs?

Cinq revues de notre corpus ont un facteur d'impact de l'ISI (2009). Deux font partie du noyau dur du domaine ICD (cf. tableau 7).

\begin{tabular}{|l|l|c|c|}
\hline \multicolumn{1}{|c|}{ Titre } & \multicolumn{1}{|c|}{ Editeur } & Nb Citations & \multicolumn{1}{c|}{ FI } \\
\hline Revue internationale de psychologie sociale & Presses universitaires de Grenoble & 86 & 0.341 \\
\hline Annales des Télécommunications & Springer France & 232 & 0.325 \\
\hline Revue française de sociologie & Ophrys & 281 & 0.263 \\
\hline Sociologie du travail & Elsevier BV & 160 & 0.340 \\
\hline Canadian Journal of Information and Library Science & University of Toronto Press & 60 & 0.000 \\
\hline
\end{tabular}

Tableau 7 : Présence d'un facteur d'impact ISI (2009)

Ces revues totalisent 819 citations en 2009. Le niveau des valeurs du facteur d'impact est relativement bas $(<0,5)$.

Douze revues du corpus sont indexées dans Scopus et ont un facteur d'impact SJR de SCImago (2009). Tandis que la Revue internationale de psychologie sociale arrive de nouveau en tête du classement, on y trouve davantage de titres cœur ICD (cf. tableau 8).

\footnotetext{
${ }^{10}$ Pour plus de détails sur ces deux indicateurs et la critique, cf. [23].
} 


\begin{tabular}{|l|l|c|}
\hline \multicolumn{1}{|c|}{ Titre } & \multicolumn{1}{c|}{ Editeur } & SJR \\
\hline Revue internationale de psychologie sociale & Presses universitaires de Grenoble & 0.048 \\
\hline Annales des Télécommunications & Springer France & 0.039 \\
\hline Réseaux: communication technologie société & La Découverte & 0.036 \\
\hline Revue française de sociologie & Ophrys & 0.035 \\
\hline Sociologie du travail & Elsevier BV & 0.035 \\
\hline Document numérique & Lavoisier & 0.034 \\
\hline Documentaliste & Association française des documentalis & 0.034 \\
\hline Etudes Photographiques & La Société Française de Photographie & 0.034 \\
\hline Le Temps Des Médias & Nouveau Monde Editions & 0.034 \\
\hline Meta : Journal Des Traducteurs & Presses de l'Université de Montréal & 0.034 \\
\hline Scriptorium & Centre d'Etude des Manuscrits & 0.034 \\
\hline Positif & Scope Editions & 0.033 \\
\hline
\end{tabular}

Tableau 8 : Présence d'un facteur d'impact SJR (2009)

La comparaison des deux tableaux révèle que même si 13 titres ont l'un ou l'autre des deux indicateurs (12\%), seulement quatre sont référencés par les deux outils scientométriques (tableau 9).

\begin{tabular}{|l|l|c|c|}
\hline \multicolumn{1}{|c|}{ Titre } & \multicolumn{1}{c|}{ Editeur } & \multicolumn{1}{c|}{ SJR 2009 IF 2009 } \\
\hline Revue internationale de psychologie sociale & Presses universitaires de Grenoble & 0.048 & 0.341 \\
\hline Sociologie du travail & Elsevier BV & 0.035 & 0.340 \\
\hline Annales des Télécommunications & Springer France & 0.039 & 0.325 \\
\hline Revue française de sociologie & Ophrys & 0.035 & 0.263 \\
\hline
\end{tabular}

Tableau 9 : Présence des deux facteurs d'impact ISI et SJR (2009)

On peut interpréter ce constat comme inadéquation des outils scientométriques, et/ou comme problème de qualité et/ou de référencement des revues.

\section{Discussion}

\section{Difficultés méthodologiques}

L'exploitation de onze sources différentes induit des problèmes de doublons, d'indexation (hétérogénéité), de terminologie (langue, nomenclature) et d'exhaustivité (données incomplètes ou manquantes). Dassa et al. [6] ont décrit les difficultés à établir une classification par discipline et des correspondances entre les nomenclatures des sources.

Un autre problème est lié au marché de l'édition avec de nombreux changements de titres ou d'éditeurs, avec des fusions, suspensions ou cessations. La différence de date des sources introduit un biais de temporalité. Même si l'information des bases de données est généralement fiable, il restera toujours un risque d'erreur ou d'imprécision. ${ }^{11}$

\section{Au-delà du domaine ICD}

Les résultats de notre analyse posent plusieurs questions. Le point de départ est une compilation et une mise en correspondance de périodiques indexés comme revues scientifiques appartenant au domaine ICD. Au final, un corpus hétérogène composé de 106 titres scientifiques et professionnels, avec ou sans peer review, issus de plusieurs domaines de recherche.

Sous l'angle des revues, ce champ de recherche se révèle comme une discipline composite, au carrefour entre recherche et métier, fruit d'échanges avec d'autres disciplines mais aussi d'une histoire particulière, construite, revendiquée, assumée [3]. «Les sciences de

\footnotetext{
${ }^{11}$ Par exemple, par rapport à l'écriture du titre et de l'éditeur.
} 
l'information et de la communication ne sont sans doute pas caractérisées autant que d'autres disciplines par un noyau dur de revues centrales et essentielles » [7].

Les liens organiques et historiques des SIC avec formations, techniques et métiers ont été analysés par Miège [17]. La proximité avec les professionnels de l'information et de la communication dépasse le concept d'une recherche appliquée et implique qu'une contribution scientifique est attendue de ces professionnels plus à l'aise pour appréhender les différents outils bibliométriques. Peut-être faudrait-il aussi transcender l'idée de la communauté scientifique pour l'ouvrir vers le milieu professionnel. Il y a quinze ans, Couzinet avait déjà étudié ce caractère hybride du format et du contenu des articles de la revue DocumentalisteSciences de l'Information [4][5].

Quant à l'interdisciplinarité, notre corpus confirme au niveau des revues ce qu'une recherche dans les bases de données scientométriques ${ }^{12}$ montre au niveau des articles: contrairement à d'autres disciplines, une partie significative des publications du domaine ICD est diffusée dans des revues multidisciplinaires ou appartenant à d'autres disciplines. C'est un fait, ce n'est pas un problème en soi. On peut néanmoins se demander quel est l'impact sur la visibilité du domaine ICD et aussi sur le mode de production scientifique, dans la mesure où chaque discipline a ses propres habitudes, modalités et contraintes.

\section{Francophonie et internationalité}

Le focus de cette étude se concentre sur les revues éditées en France et/ou de langue française. Dans le milieu de l'édition scientifique, on compte en moyenne 50-100 auteurs réguliers par revue. Le Ministère de l'Enseignement Supérieur et de la Recherche chiffre la communauté des SIC en France à 600-700 chercheurs [24]. Il y aurait donc de la place pour l'édition d'au moins 5-15 périodiques. Ce chiffre correspond assez bien au nombre des « revues cœur ».

Le nombre plus élevé de l'ensemble du corpus s'expliquera assez facilement par la transversalité et l'interdisciplinarité du domaine ICD, animées par des communautés mixtes dépassant les équipes de chercheurs avec un nombre important de professionnels de l'information et de la communication. D'autre part, il faut tenir compte de la présence de communautés francophones belge et canadienne.

A ce jour, il n'existe pas en France de base de données ou d'archive qui recenserait les publications du domaine ICD d'une façon exhaustive ${ }^{13}$. Il est difficile à chiffrer la production annuelle d'articles dans ces revues. Cependant, une extrapolation à partir de FRANCIS permet d'estimer leur potentiel annuel à environ 2000 articles de tout genre. Ce n'est pas rien.

Et pourtant - même s'il parait exagéré de parler d'une tendance à la ghettoïsation [16], le manque d'impact et de visibilité sur la scène internationale est significatif. Cette situation freine le développement de coopérations ou partenariats et pénalise l'évaluation et l'octroi de subvention (ANR, FP7 etc.) car la recherche internationale devient progressivement un critère prioritaire.

Certes, cette situation n'est pas propre au domaine ICD. Mais les publications d'autres disciplines - et pas seulement en sciences médicales ou sciences de la vie - sont mieux représentées dans les outils scientométriques. En ce qui concerne les SHS en France, les

\footnotetext{
${ }^{12}$ Un exemple (juin 2011) : en recherchant les articles indexés sous le mot-clé scientometrics, Scopus identifie 222 références publiées depuis 2000. Parmi ces références, 114 articles $(51,4 \%)$ sont publiés dans des revues en dehors du champ ICD. Ces articles totalisent $57 \%$ des citations.

${ }^{13}$ Créée en 2002, l'entrepôt ArchiveSIC répertorie les publications des chercheurs et professionnels en SIC déposées sur la base du volontariat. ArchiveSIC contient 1700 références dont $80 \%$ avec accès au plein texte.
} 
revues en psychologie, sociologie ou économie ont par exemple plus d'impact et de visibilité que celles des SIC [23].

Cette relative invisibilité sur la scène internationale est une difficulté qui ne se réduit pas à une question de barrière linguistique [11]. D'autres facteurs sont en jeu, comme par exemple une politique éditoriale claire et affichée en anglais, un mode (et un taux) de sélection explicite et conforme aux standards internationaux, une traduction des titres, résumés et informations pour les auteurs, un comité de rédaction international, une périodicité régulière et souvent plus élevée, ou une stratégie de promotion, de communication et de référencement. De plus, les définitions disciplinaires des SIC francophones, LIS anglophones, Bibliothekswissenschaften allemandes etc. ne se recoupent pas tout à fait ce qui complique la réception, le référencement et la visibilité au plan international.

Le lien entre communauté, francophonie et internationalité est là : le développement de l'impact international du domaine ICD francophone ne passe pas nécessairement ni uniquement par l'abandon du français au profit de l'anglais, mais aussi par le développement de la qualité des revues. Pour reprendre la remarque de Garfield [9] citée plus haut : ces revues francophones pourraient avoir le même degré d'impact que les revues internationales à condition d'adopter les mêmes règles que les titres phares et d'attirer d'importants travaux de recherche originaux.

\section{Conclusion}

L'article débute par le constat que la revue scientifique joue un rôle central dans la communication scientifique, pour la diffusion des résultats et pour l'accès aux connaissances. Riche d'une histoire de plusieurs siècles, la revue scientifique continue à refléter et façonner le mode de fonctionnement d'une communauté scientifique, y compris l'évaluation.

Une étude empirique sur les revues d'une discipline parlera toujours de la discipline ellemême, dans la mesure où toute réflexion sur la discipline portera aussi sur les modalités d'acquisition, de production et de diffusion des connaissances.

L'image qui se dégage de notre analyse est contrastée. Le référencement des périodiques francophones renvoie l'image d'une discipline assez hétérogène, interdisciplinaire, transversale, dont les contours ne sont pas toujours clairs. Une autre caractéristique se définit par une forte contribution professionnelle mais sans réelle visibilité ni impact significatif sur la scène internationale, en dehors de la francophonie.

Le trait est certainement forcé. En particulier, l'analyse ne tient pas compte de la partie non francophone de la production scientifique qui contribue au rayonnement du domaine ICD français. Mais l'analyse confirme que le cœur (et a fortiori, la périphérie) des revues de cette communauté reste en grande partie en dehors ou en-deçà des publications de renommée internationale.

Pour conclure, trois pistes d'études :

(1) Une des particularités de cette analyse est que son corpus fait partie d'un ensemble de 1218 périodiques ICD sur le plan international. Il est donc tout à fait possible et envisageable d'effectuer une comparaison entre les 106 revues francophones et les autres afin d'étudier de plus près les liens entre francophonie, internationalité et communauté.

(2) Cette analyse a adopté une approche assez large, sans limiter le corpus à quelques titres reconnus. Or, il serait intéressant de revenir sur le concept du caractère scientifique d'un périodique ICD, d'analyser ce qui contribue à sa reconnaissance et à sa réputation. Ceci 
pourrait se faire en appliquant les approches qualitatives de Jeannin [14] et Schloegl \& Stock [21]. A priori, il paraît possible d'en dériver un indicateur quantitatif.

(3) Nous n'avons pris en compte le fait que de plus en plus de travaux sont publiés en dehors du système traditionnel de l'édition, en particulier dans des archives ouvertes. Même si les périodiques ICD en libre accès (avec ou sans embargo) restent encore une minorité, un nombre croissant de publications est déposé dans une ou plusieurs archives ouvertes. Un document sur six déposé dans ArchiveSIC n'est pas publié ailleurs. Même si pour l'instant il s'agit d'un choix individuel sans concurrence au système traditionnel car ces dépôts ne sont pas évalués, il est tout de même certain qu'ils ont un impact et une visibilité intéressante [15]. Pouchot \& Prime-Claverie [19] viennent de publier une première étude bibliométrique à ce propos, dont les résultats et perspectives sont prometteurs.

Le besoin fait l'outil, et l'outil façonne l'usage. La transformation en profondeur des modes de production et de distribution des revues scientifiques et l'émergence de nouveaux formats, contenus et services détermineront à terme aussi les méthodes de recherche, la circulation des savoirs et la production des connaissances [18]. Les sciences de l'information et de la communication progresseront aussi grâce à une évaluation constante et une maîtrise accrue de leurs outils et vecteurs de communication.

\section{Bibliographie}

[1] Judith BAR-ILAN. 'Rankings of information and library science journals by JIF and by h-type indices'. Journal of Informetrics, 2010, vol 4, n², p.141-147.

[2] Guylaine BEAUDRY. La communication scientifique et le numérique. Paris: Lavoisier, 2011. 327 p.

[3] Robert BOURE. (dir.). Les origines des sciences de l'information et de la communication - regards croisés. Villeneuve d'Ascq : Presses Universitaires Du Septentrion, 2002. 184 p.

[4] Viviane COUZINET. 'De l'information professionnelle à l'information scientifique. Quelle place pour la recherche dans la revue Documentaliste-Sciences de l'information ?'. Documentaliste - Sciences de l'Information, 1997, vol 34, n³, p.147-154. (a)

[5] Viviane COUZINET. 'Pratiques professionnelles, pratiques de recherche. Les articles de la revue Documentaliste-Sciences de l'information'. Documentaliste - Sciences de l'Information, vol.34, ${ }^{\circ}$ 6, p.289-299. (b)

[6] Michèle DASSA, Christine KOSMOPOULOS et Denise PUMAIN 'JournalBase. Comparer les bases de données scientifiques internationales en sciences humaines et sociales (SHS)'. Cybergeo : European Journal of Geography, 2010.

http://cybergeo.revues.org/22864

[7] Béatrice FLEURY et Jacques WALTER. 'L'histoire des sciences de l'information et de la communication'. Questions de Communication, 2007, n²12, p.133-148.

[8] Einar H. FREDRIKSSON (dir.). A century of science publishing. [s.1], IOS Press, 2001. 312 p.

[9] Eugène GARFIELD. 'Citation consciousness. The origins of citation indexing in science (Interview with Wolfgang G. Stock)'. Password, 2002, n ${ }^{\circ}$, p.22-25. http://www.garfield.library.upenn.edu/papers/passwordinterview062002.pdf

[10] GFII. 'L'édition scientifique française en sciences sociales et humaines'. Rapport de synthèse, Paris, novembre 2009. 42 p. 
http://www.gfii.fr/uploads/docs/l-edition-scientifique-francaise-en-sciences-sociales-et$\underline{\text { humaines.pdf }}$

[11] François HEINDERYCKX, Margaux HARDY et Marc VANHOLSBEECK. 'Les revues scientifiques en information-communication'. Questions de Communication, 2012 (à paraître).

[12] Chang-Ping HU et al. 'A journal co-citation analysis of library and information science in China'. Scientometrics , 2011, vol 86, n³, p.657-670.

http://www.akademiai.com/content/m7n221w83h61mt55/fulltext.pdf

[13] Philippe JEANNIN. `Revuemétrie de la recherche en sciences humaines et sociales. Rapport synthétique et final de mission (1999-2003)'. Paris, Direction de la Recherche. Ministère délégué à la recherche et aux nouvelles technologies, novembre 2003, 41 p. http://www.revues.org/cost/images/1/12/JEANNIN___Rapport_final2003a.pdf

[14] Philippe JEANNIN. 'Retour sur l'enquête (2000-2001) relative aux périodiques scientifiques en sciences de l'information et de la communication (SIC)'. In 2ème journée d'étude sur les revues en sciences de la communication, Paris, 14 mai 2009

[15] Olivier LE DEUFF `Pour une évaluation diversifiée et de nouvelles métries'. Article de blog. Le guide des égarés, 12 mai 2010.

http://www.guidedesegares.info/2010/05/12/pour-une-evaluation-diversifiee-et-de-nouvelles$\underline{\text { metries/ }}$

[16] Annie Le SAULX. 'Les revues en sciences de la communication'. Bulletin des Bibliothèques de France, 2009, vol 54, n²4, p.101-102.

http://bbf.enssib.fr/consulter/bbf-2009-04-0101-007

[17] Bernard MIEGE. 'Les apports à la recherche des sciences de l'information et de la communication'. Réseaux, 2000, vol.18, n¹00, p.547-568.

[18] Bernard MIEGE. 'La circulation des savoirs en sciences de l'information et de la communication'. Questions de Communication, 2006, nº 9 , p.401-417.

[19] Stéphanie POUCHOT et Camille PRIME-CLAVERIE. 'Quelles images bibliométriques de la recherche française en SIC?'. Documentaliste, 2011, vol 48, n², p.6473.

http://hal.archives-ouvertes.fr/hal-00619035/fr/

[20] Paul RASSE et al. 'Panorama du paysage des revues de sciences de l'information et de la communication avant qu'il ne change'. Communication, 2009, vol 27, n², p.217-233.

[21] Christian SCHLOEGL et Wolfgang G. STOCK. 'Impact and Relevance of LIS Journals: A Scientometric Analysis of International and German-Language LIS Journals Citation Analysis Versus Reader Survey'. Journal of the American Society for Information Science and Technology, 2004, vol 55, n¹3, p.1155-1168.

[22] Christian SCHLOEGL et Wolfgang PETSCHNIG. 'Library and information science journals: An editor survey'. Library Collections, Acquisitions, and Technical Services, 2005, vol $29, \mathrm{n}^{\circ} 1, \mathrm{p} .4-32$.

http://www.kfunigraz.ac.at/iwiwww/publ/schloegl_2005a.pdf

[23] Joachim SCHÖPFEL et Hélène PROST. 'Le JCR facteur d'impact (IF) et le SCImago Journal Rank Indicator (SJR) des revues françaises : une étude comparative'. La Psychologie Française, 2009, vol 54, nº 4 , p. 287-305.

http://archivesic.ccsd.cnrs.fr/sic_00567847_v1/ 
[24] SNRI. 'Nouvelle nomenclature des Sciences de l'Homme et de la Société'. Rapport, Stratégie Nationale de Recherche et d'Innovation. Paris, Ministère de l'Enseignement Supérieur et de la Recherche, décembre 2010, 11 p.

http://media.enseignementsup-

recherche.gouv.fr/file/Innovation,_recherche_et_developpement_economique/02/8/Nomencla ture_SHS_167028.pdf

[25] Anthony F. J. VAN RAAN, Thed N. VAN LEEUWEN et Martijn S. VISSER 'Severe language effect in university rankings: particularly Germany and France are wronged in citation-based rankings'. Scientometrics, 2011, vol 88, n², p.495-498.

http://arxiv.org/abs/1012.5199

[26] Dangzhi ZHAO. 'Characteristics and impact of grant-funded research: a case study of the library and information science field'. Scientometrics, 2010, vol 84, n², p.293-306.

http://www.springerlink.com/content/0681u2280t22t104/

Nous remercions Chérifa Boukacem, Etienne Fleuret, Hakim Harik, Philippe Jeannin et trois relecteurs anonymes pour leur conseils et commentaires utiles. 